Rev. Biol. Trop. 52(1): 31-40, 2004

www.ucr.ac.cr www.ots.ac.cr www.ots.duke.edu

\title{
Descripción ultraestructural de Euglena pailasensis (Euglenozoa) del Volcán Rincón de la Vieja, Guanacaste, Costa Rica
}

\author{
Ethel Sánchez, ${ }_{1}^{1}$ Maribel Vargas, ${ }^{1}$ Marielos Mora, ${ }^{2}$ José María Ortega, ${ }^{3}$ Aurelio Serrano, ${ }^{3}$ \\ Enrique Freer ${ }^{1} \&$ Ana Sittenfeld ${ }^{2}$ \\ 1 Centro de Investigación en Estructuras Microscópicas, Universidad de Costa Rica, Apdo. 2060 San Pedro, Montes de \\ Oca, San José, Costa Rica. Fax (506)207-3182; ethels@cariari.ucr.ac.cr \\ 2 Centro de Investigación en Biología Celular y Molecular, Universidad de Costa Rica, San Pedro de Montes de Oca, \\ San José, Costa Rica. Fax (506) 207-3190. \\ 3 Instituto de Bioquímica Vegetal y Fotosíntesis (IBVF), Universidad de Sevilla y CSIC, Américo Vespucio s/n, 41092, \\ España.
}

Recibido 02-III-2002. Corregido 22-III-2003. Aceptado 23-IV-2003.

\begin{abstract}
The euglenoids are unicellular eukaryotic flagellates living in a diversity of soils and aquatic environments and ecosystems. This study describes the ultrastructure of an euglenoid isolated from the surface of a boiling mud pool with temperatures ranging from 38 to $98^{\circ} \mathrm{C}$ and $\mathrm{pH} 2-4$. The hot mud pool is located in Area de Pailas de Barro, Las Pailas, Rincón de la Vieja Volcano, Guanacaste, Costa Rica. The morphological characterization of the Euglena pailasensis was performed by SEM and TEM. It was determined that, although the euglenoid was obtained from an extreme volcanic environment, the general morphology corresponds to that of a typical member of Euglena of 30-45 $\mu \mathrm{m}$ long and 8-10 $\mu \mathrm{m}$ wide, with membrane, pellicle, chloroplasts, mitochondria, nucleus, pigments and other cytoplasmic organelles. E. pailasensis is delimited by a membrane and by 40 to 90 pellicle strips. It was observed up to 5 elongated chloroplasts per cell. The chloroplast contains several osmiophilic globules and a pyrenoid penetrated by few thylakoid pairs. The nutritious material is reserved in numerous small paramylon grains located at the center of the cell, mitocondria are characterized by the presence of crests in radial disposition toward the interior of the lumen. It was also observed around the external surface "pili" like filaments originating from the pellicle strips. There is no evidence for the presence of flagella in the ampulla (reservoir/canal area), a fact confirmed by negative staining, and a difference regarding other species of Euglena. The observed ultrastructural characteristics are not sufficient to explain the adaptation of this species to acid and hot environments.
\end{abstract}

Key words: Protozoa, Euglenozoa, euglenoid, Euglena, ultrastructure, termoresistent, acid tolerant.

Los euglenoides pertenecen a la filo Euglenozoa, clase Euglenida. Son microorganismos unicelulares eucariotas, que habitan en suelos y en una diversidad de ambientes acuáticos, tanto dulceacuícolas como marinos (Müllner et al. 2001). La mayoría tienen dos flagelos anteriores, uno de los cuales es emergente (Leedale et al.1965) y su tamaño varía entre $10 \mu \mathrm{m}$ y $500 \mu \mathrm{m}$ de longitud (Mermelstein et al. 1998).
El género Euglena se distingue de otros protistas por presentar características propias como la presencia de cloroplastos y la permanencia del nucleolo y endosomas durante la división mitótica (Leedale et al. 1965, Leedale 1967, Kempner y Marr 1970, Van den Hoek et al. 1993, Wen 2000). Es evidente la presencia de vacuolas de reserva compuestas por paramilón (beta- 1:3 glucopiranósido) (Leedale et al. 1965, Schawartzbach et al. 1975, Osafune et 
al. 1990, Van den Hoek et al. 1993). Externamente la célula presenta una película proteica flexible formada por mionemas (Leedale 1964, Leedale et al. 1965, Summer 1965, Van den Hoek et al. 1993, Arnott y Farmer 2000, Vismara et al. 2000). Un flagelo activo y corto emerge desde un complejo reservorio sub-apical. Un segundo flagelo no emergente está presente en el reservorio. Las células no son rígidas y se desplazan por medio del movimiento euglenoide conocido como metabolia. Sus cloroplastos desaparecen cuando se cultivan en oscuridad, reapareciendo una vez que se vuelven a cultivar bajo condiciones de luz (Schwartzbach et al. 1975, Osafune et al. 1990).

Desde la descripción del género Euglena por Ehrenberg (1838) (Pringsheim 1948), se han descrito más de 150 especies cuya división taxonómica no ha sido concluyente; sin embargo, los estudios genéticos basados en análisis del ADNr 18S brindan una nueva base para su clasificación (Müllner et al. 2001).

Estudios realizados (Brock 1986, Walsh y Seckbach 1999, Sittenfeld et al. 2002) para evaluar la presencia de microorganismos fotosintéticos adaptados a condiciones extremas como alta temperatura y $\mathrm{pH}$ ácidos en rocas, lodos ácidos y aguas termales del volcán Rincón de la Vieja (Area de Conservación Guanacaste, noroeste de Costa Rica) demostraron la presencia de un euglenoide (CRRdV) en parches verdes sobre la superficie del lodo caliente de una de las pailas ( $\mathrm{pH} 2-4 ; 35-98^{\circ} \mathrm{C}$ ) localizadas en el sector de Las Pailas de Barro Caliente, situado en la ladera sur del cráter principal. Los estudios fisiológicos y filogenéticos revelaron que éste es capaz de crecer en medio mineral definido a temperaturas hasta de $40^{\circ} \mathrm{C}$ con una termotolerancia en su actividad fotosintética mayor que la cepa tipo de Euglena gracilis SAG 5/15, así como una viabilidad de 1-2 horas a $50^{\circ} \mathrm{C}$ en las condiciones naturales del sitio de estudio. El euglenoide que ha sido recientemente descrito como Euglena pailasensis Sittenfeld et al. (2004), comparte una similitud de $51.1 \%$ con E. mutabilis en una matriz de identidad basada en la secuencia del ADNr 18S (Sittenfeld et al. 2002).
Debido a las características del ambiente (alta temperatura y $\mathrm{pH}$ ácido) en que habita E. pailasensis pareció conveniente realizar un detallado estudio morfológico ultraestructural de este organismo.

\section{MATERIALES Y MÉTODOS}

Se recolectaron muestras de barro y de parches verdes en la superficie del lodo caliente de una de las fuentes en el área conocida como Las Pailas de Barro Caliente, del Volcán Rincón de la Vieja, ubicado en el Parque Nacional Rincón de la Vieja, Guanacaste, Costa Rica. El material de los parches verdes, correspondió a una población aparentemente unialgal de E. pailasensis. Los ejemplares fueron aislados y cultivados en medio mineral (Sueoka et al. 1967). Alícuotas de $1 \mathrm{ml}$ de las muestras de barro y de los cultivos se concentraron por ultracentrifugación para el análisis ultraestructural y se fijaron en solución de glutaraldehido al $2.5 \%$ y paraformaldehído al $2 \%$ en amortiguador de fosfato de sodio $(0.1 \mathrm{M} \mathrm{pH}$ 7.4), se lavó y posfijó por 1 hora en tetraóxido de osmio al $1 \%$ diluido en el amortiguador y luego se lavó y continuó el procesamiento para microscopia electrónica.

Un volumen de $0.5 \mathrm{ml}$ de muestra se incluyó en agar al 3\%. Los bloques de agar se seccionaron en trozos de $3 \mathrm{~mm}^{3}$, se deshidrataron utilizando un gradiente ascendente de alcohol etílico $(30 \%$ a $100 \%)$ e infiltraron con resina Spuur. Se polimerizaron y se hicieron cortes de 70 y $80 \mathrm{~nm}$ de grosor, los cuales se contrastaron con acetato de uranilo e hidróxido de plomo y se observaron con microscopios electrónicos de transmisión H-7000 y H-7100 (Hitachi) a un voltaje de aceleración de $100 \mathrm{KV}$.

Un volumen de $0.1 \mathrm{ml}$ de las muestras se impregnó en rejillas recubiertas con membrana de formvar, se sometieron a tinción negativa con ácido fosfotúngstico $(0.5 \% \mathrm{pH} 7)$ y se observaron con el microscopio electrónico de transmisión H-7100.

Posteriormente estas rejillas fueron montadas en bases de aluminio y se cubrieron con 
una capa de oro de $20 \mathrm{~nm}$ de espesor y se observaron con microscopios electrónicos de barrido S-570 y S-2360N (Hitachi).

\section{RESULTADOS}

La morfología general del E. pailasensis es la de una célula típica del género Euglena, con un tamaño que varía entre 30 - $45 \mu \mathrm{m}$ de largo y 8-10 $\mu \mathrm{m}$ de ancho y su forma puede ser muy variada, desde muy esférica hasta muy elongada (Figs. 1a y 1b).

La membrana citoplasmática, constituida por una doble membrana, es continua y cubre por completo la película (Fig. 2a). La película es una estructura compuesta por bandas planas que están entrelazadas dispuestas helicoidalmente sobre toda la célula y que alternan con una franja, hendidura o surco de naturaleza más suave (Figs. 1a y 1c). Estas bandas o mionemas tienen un grosor aproximado entre $0.8 \mathrm{y}$ $1.0 \mu \mathrm{m}$, son semi-rígidas y una célula puede tener entre 40 y 90 mionemas. La película está rodeada por filamentos muy finos semejantes a "pili" (pelos) (Fig. 1d), pero no se observaron flagelos ni estructuras asociadas a estos, tanto en las alícuotas obtenidas a partir del barro como a partir de cultivos en medio mineral. Cuando se observa la célula viva con el microscopio de luz, se nota que las bandas de la película se mueven una contra la otra.

En la figura $2 \mathrm{a}$, se observa canales del retículo endoplásmico y microtúbulos paralelos a lo largo de cada mionema de la película, estos microtúbulos tienen un diámetro aproximado entre 200 y $250 \AA$ A . Tres de ellos siempre se observan muy juntos e inmediatamente adyacentes a la membrana en la hendidura o surco.

En la figura $2 b$ se observa el núcleo, que puede presentar forma ovoide $\mathrm{u}$ ocasionalmente esférica, está limitado por la envoltura nuclear y durante la interfase se localiza centralmente y los cromosomas se observan contraídos. La envoltura nuclear es doble y consiste de dos membranas contorneadas irregularmente y constituyen la unidad de membrana, que a su vez están separadas por un pequeño espacio. El nucleolo es ovoide grande y denso y por lo general se localiza hacia el centro del núcleo. Los cromosomas, el nucleolo y el nucleoplasma se observan como áreas uniformemente granulares de diferente densidad electrónica (Figs. 2b y 2c).

Hacia el interior celular, pero cerca de la película, se observan las cisternas tubulares del retículo endoplásmico (Figs. 2a y 2d), que es un complejo citoplasmático compuesto de vesículas y túbulos. Las membranas de retículo endoplásmico tubular se observan lisas mientras que otras son rugosas y presentan ribosomas asociados a su membrana. Este complejo membranoso constituye un sistema de interconexiones a través de toda la célula (Fig. 2d).

E. pailasensis presenta varios aparatos de Golgi o dictiosomas distribuidos a través de la matriz citoplasmática y cada uno de ellos puede medir entre 0.5 y $1 \mathrm{~mm}$ de longitud. Aparentemente estos dictiosomas son muy similares a los de otras células eucariotas, compuestos por un largo cilindro apilado y curvado con cisternas aplanadas. Las cisternas están conectadas a intervalos y parece que forman pequeñas vesículas (50-70 nm de diámetro) desde su borde fenestrado (Fig. 2d).

Las mitocondrias por lo general son de forma ovoide o alargadas, miden aproximadamente $360 \mathrm{~nm}$ de ancho por $675 \mathrm{~nm}$ de largo y se encuentran dispersas a través de la matriz citoplasmática. La membrana externa se nota marcadamente ondulante en ciertas secciones y la membrana interna forma las crestas que se notan orientadas radialmente hacia el interior de la matriz del lumen mitocondrial (Fig. 3a).

En los cortes ultrafinos se observó hasta cinco cloroplastos por célula, ubicados usualmente cerca de la periferia celular. Los cloroplastos son elongados, de forma más o menos cilíndrica y pueden medir aproximadamente de 1 a $2 \mu \mathrm{m}$ de diámetro y de 6 a $12 \mu \mathrm{m}$ de largo (Figs. 2c, 3b y 3c), cada uno de ellos está delimitado por tres membranas, seguida por membranas paralelas o lamelas que constituyen los tilacoides, localizados en el estroma (Fig. 3b). Entre las lamelas se encontraron glóbulos osmiofílicos, que se contrastan intensamente con 


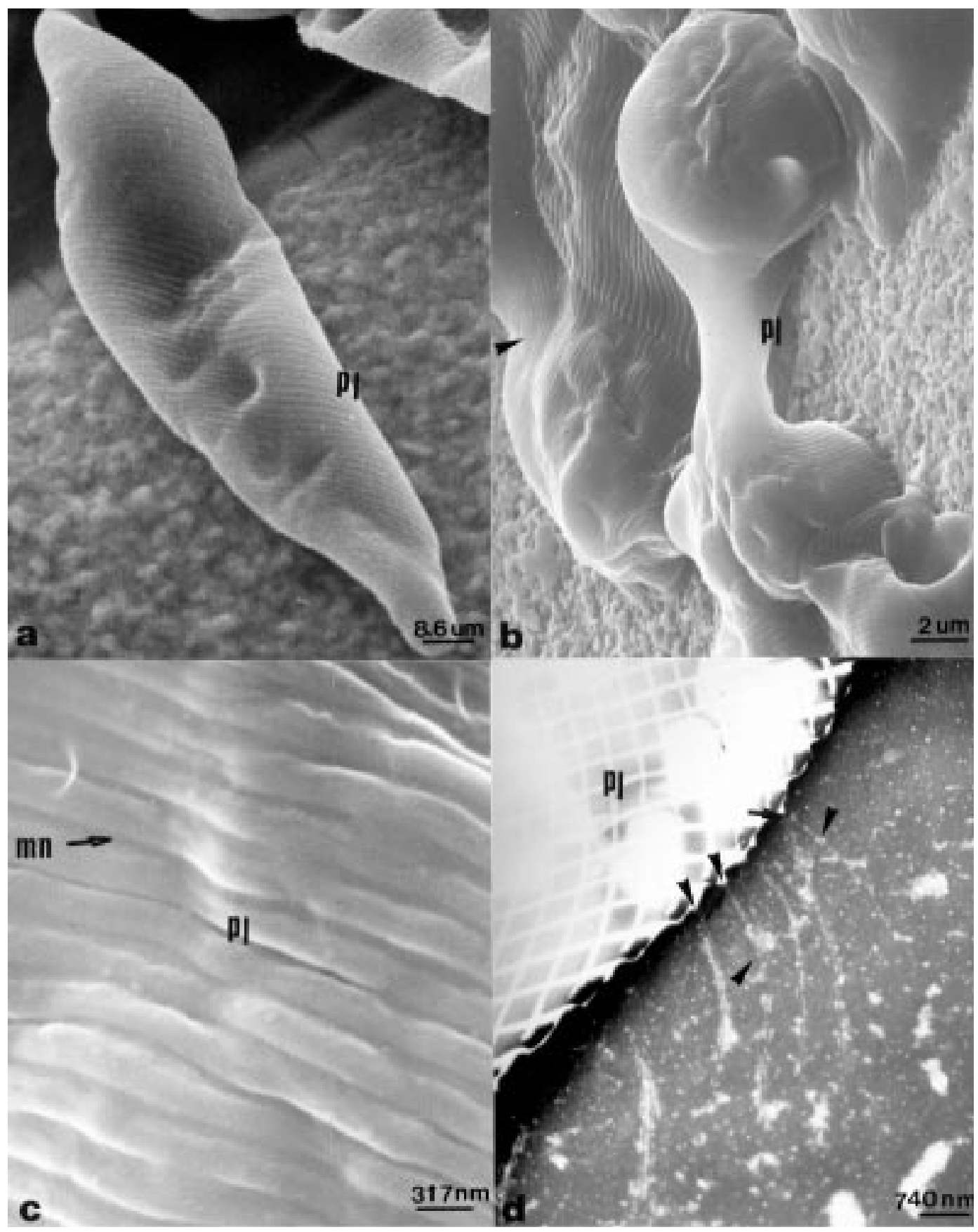

Fig. 1. a) E. pailasensis elongado, se muestra el recubrimiento helicoidal de la película. b) Especímenes cubiertos por material mucilaginoso (flecha). c) Detalle de la película (pl) mostrando los mionemas. d) Se observan los "pili" o filamentos asociados a los mionemas (flechas).

Fig. 1. a) Elongated E. pailasensis covered with a helicoidally shaped pellicle. b) Specimens covered by a muscilagous material (arrow). c) A detail of the pellicle (PI) showing the mionemas. d) Pili or filaments associated to the mionemas are observed (arrows). 


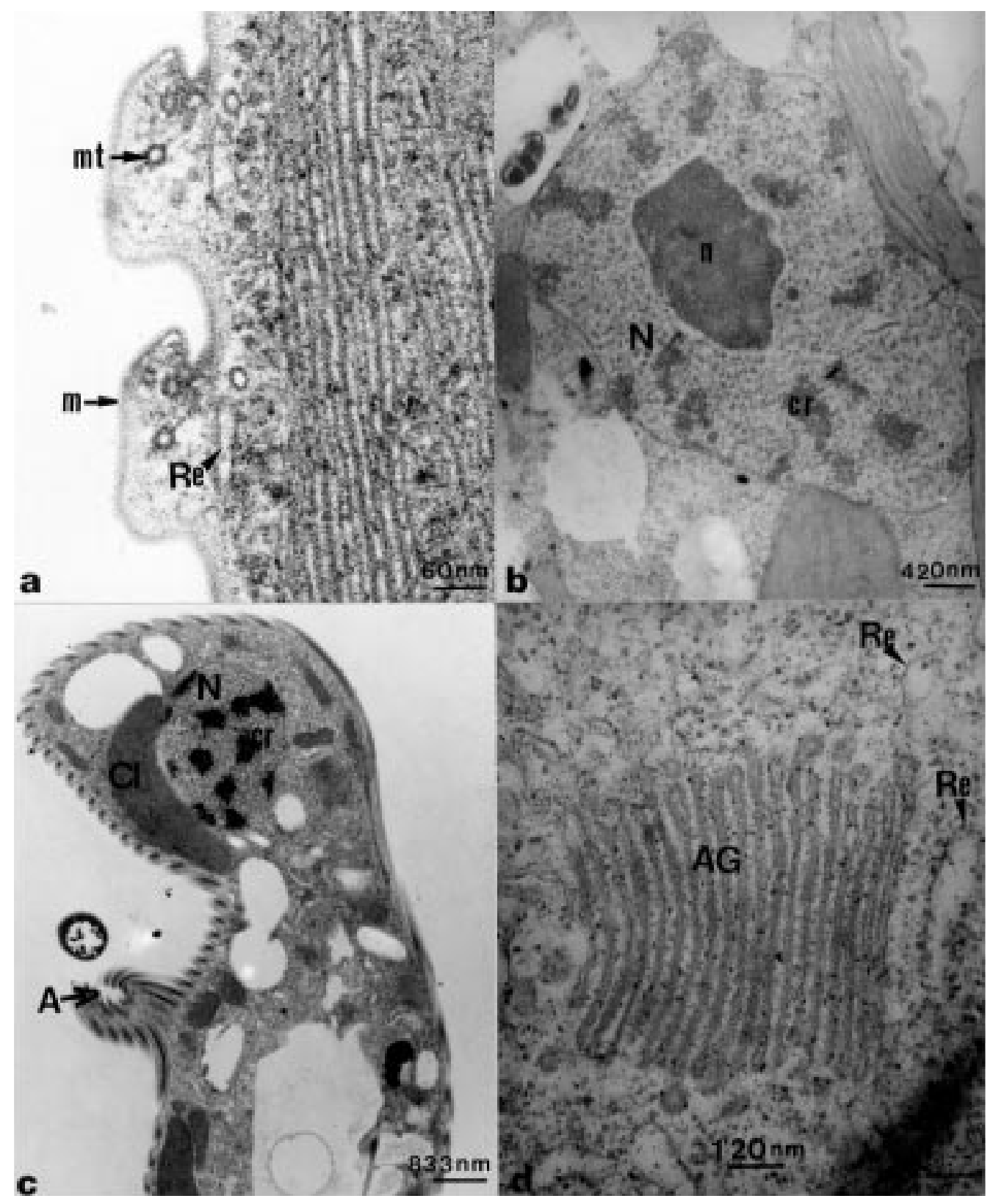

Fig. 2. a) Se observa la membrana plasmática recubriendo los mionemas (m) de la película. También se observa los microtúbulos (mt) y secciones del retículo endoplásmico tubular (Re). b) El núcleo (N) en posición central, el nucleolo (n) y los cromosomas (cr) contraídos. c) Corte longitudinal de un euglenoide, se observa la ámpula (A), en aparente proceso de ingestión de alimento. d) En el citoplasma se observa el aparato de Golgi (AG) y varias vesículas del retículo endoplásmico tubular $(\operatorname{Re})$.

Fig. 2. a) The plasmatic membrane is observed covering the pelicle mionemas (m). Also, microtubules (mt) are observed and sections of the tubular endoplasmic reticulum (Re). b). The nucleus $(\mathrm{N})$ is in central position, nucleolus (n) and chromosomes (cr) are contracted. c).The cytoplasm contains the Golgi Apparatus (AG) and several vesicles from the tubular encoplasmic reticulum. 
el tetróxido de osmio y que pueden medir entre 50 y 300 nm de diámetro (Fig. 3c), también observamos en algunos cloroplastos un pirenoide, localizado centralmente en el plastidio, la matriz del pirenoide es penetrada por pocos tilacoides que corren continuos a las típicas tripletas lamelares del euglenoide (Fig. 3c).

Es característica del género Euglena la presencia de una ámpula, una invaginación en la parte anterior de la célula diferenciada en un canal y un reservorio. Esta invaginación en E. pailasensis consiste también en un canal y un reservorio que pueden variar de forma y tamaño dependiendo del movimiento de la célula y las sustancias a ingerir (Fig. 3d). Fue frecuente observar en esta estructura invaginaciones de la película que sugieren que aunque este euglenoide debe ser autótrofo, podría ingerir también algún tipo de alimento (Figs. 2c y $3 d$ ). No se observaron flagelos ni estructuras paraflagelares dentro del ámpula.

En el citoplasma se notó la presencia de estructuras constituidas por vesículas de varias formas y tamaños, que presentan una proliferación de unidades de membranas que se observan muy electrodensas y que son también llamadas pirenoides (Fig. 4a).

El material nutritivo se almacena en forma de granos de paramilón. En un corte longitudinal del ejemplar fue posible contar hasta 75 gránulos, que son pequeños, de forma bacilar y discoidal, localizados en toda la célula y menos electrodensos que los pirenoides (Fig. 4b).

También se observó la vacuola contráctil, que cuando está llena, tiene forma esférica y por lo general en su interior se nota un número de pequeñas vacuolas accesorias (Fig. 4c).

\section{DISCUSIÓN}

Los especímenes analizados de E. pailasensis comparten las características ultraestructurales de otras especies del género Euglena (Pringsheim 1948, 1956, Leedale 1964, Leedale et al. 1965, Summer 1965, Leedale 1967, Lefort-Tran et al. 1980, Osafune et al.1990, Adachi et al. 1999, Arnott y Farmer
2000), pero no fue posible observar flagelos ni estructuras asociadas a los mismos, que caracterizan a este género (Pringsheim 1948, 1956). Tampoco se observaron flagelos con la técnica de tinción negativa (Lowy y Hanson 1965) utilizada para observar flagelos bacterianos. Sin embargo, fue posible observar el euglenoide rodeado de una serie de filamentos semejantes a "pili" (pelos) que se originan en los mionemas, característica no descrita en la literatura consultada. La identificación de esta red de fibrillas localizada en la membrana celular y que aparentemente forman el citoesqueleto de este protista, es uno de los hallazgos que aporta este trabajo al estudio de la morfología y movimiento euglenoide. Esta red de fibrillas constituye una especie de armazón que le brinda la forma, rigidez y posiblemente flexibilidad característica a este organismo. Esta estructura podría estar asociada a los mionemas, surcos de la membrana plasmática y presumiblemente actuaría a modo de soporte y órgano de movimiento, que le permite un movimiento reptante (Leedale 1964, 1967, Lefort-Tran et al. 1980, Murray 1981, Dubreuil y Bouck 1985, Van den Hoek et al. 1993, Arnott y Farmer 2000, Vismara et al. 2000).

Estudios previos, sobre la caracterización fisiológica y filogenética de E. pailasensis (Sittenfeld et al. 2002), determinaron que posee termotolerancia a temperaturas cercanas a los $45^{\circ} \mathrm{C}$ y viabilidad por varias horas a $50^{\circ} \mathrm{C}$ en el sitio de colecta. Además, está filogenéticamente relacionado con Euglena mutabilis, otro euglenoide fotosintético que tolera altos niveles de acidez (Kapfer 1998, Lessmann et al. 1999). Sittenfeld et al. (2002) mediante análisis moleculares, sugirieron que el euglenoide CRRdV (ahora E. pailasensis) correspondía a una nueva especie. Este trabajo apoya dicha sugerencia ya que: a) E. mutabilis tiene un flagelo emergente corto y E. pailasensis no; b) E. mutabilis no es termoresistente; y c) la secuencia del ADN 18S de E. pailasensis es similar en un $51.1 \%$ con la E. mutabilis, la especie más cercana en los árboles filogenéticos realizados (Sittenfeld et al. 2002). Ambas especies tienen en común la capacidad de 




Fig. 3. a) En un corte transversal del euglenoide se observan los mionemas de la película (pl) y las mitocondrias (M), se nota la orientación radial de las crestas (c). b) Se muestra un segmento de dos cloroplastos $(\mathrm{Cl})$, se nota la triple membrana $(\mathrm{mc})$ externa y los tilacoides (t) paralelos a ésta. c) En un corte transversal se aprecia el cloroplasto (Cl) elongado, con el pirenoide (P) en su interior y varios glóbulos osmiofílicos (go). d) Ámpula (A), se aprecia un adelgazamiento de la película en la parte interna.

Fig. 3. a) A transversal cut of the euglenoid shows a detail of the pellicle mionemas (pI), and the mitocondria (M) with a radial orientation of the crests (c). b) A segment of the chloroplast is showed $(\mathrm{Cl})$, an external three membrane structure is indicated (mc) and the tilakoids ( $t$ ) running parallel to the membrane. c). A transversal cut shows the elongated form of the chloroplast $(\mathrm{Cl})$, containing the pirenoid $(\mathrm{P})$ and several osmiophilic globules (go). d). Ampulla (A), showing pellicle narrowness in its internal part. 



desarrollarse en medios muy ácidos (pH 1-2). Este estudio aporta además información conducente a la descripción taxonómica del euglenoide E. pailasensis, tales como el número, tamaño y localización de los cloroplastos y gránulos de paramilón, así como la presencia de pirenoides, considerados como criterios de importancia por otros autores para la clasificación taxonómica (Zakrys et al. 2001) y que lo diferencian de E. mutabilis.

E. pailasensis podría corresponder al primer euglenoide fotosintético descrito que habita en ambientes con actividad volcánica caracterizados por $\mathrm{pH}$ bajos y altas temperaturas (Sittenfeld et al. 2002). La ausencia de flagelos y otras características ultraestructurales observadas en este estudio y que podrían diferenciar a esta especie de otros euglenoides no ofrecen, en nuestra opinión, razones suficientes para explicar la termotolerancia de E. pailasensis y su capacidad de vivir en ambientes de temperatura, $\mathrm{pH}$ y composición físico-química descritos previamente para el sitio de colecta en las Pailas de Barro Caliente.

\section{AGRADECIMIENTOS}

Los autores agradecen a los profesores Manuel Losada (Universidad de Sevilla, España), Rodrigo Gámez (Instituto Nacional de Biodiversidad, INBio, Costa Rica), Jürgen Weckesser (Universidad de Friburgo, Alemania), Mario Fernández y Francisco Hernández Chavarría (Universidad de Costa Rica, Costa

Fig. 4. a) Detalle de los pirenoides formados por un sistema de membranas electrodensas $(\mathrm{P})$. b) Gránulos de paramilón $(\mathrm{pm})$ en un corte transversal. C) Se observa la vacuola contráctil (V) con varias vacuolas accesorias internas.

Fig. 4. a) Details of the pyrenoid formed by a system of electrodense membranes (P). b) Paramylon granules (pm) in cross section. C) Contractile vacuole is observed (V) with several internal accessory vacuoles. 
Rica). Este trabajo fue posible gracias al apoyo financiero del "Plan de Cooperación Científica con Iberoamérica" 1998-2000 (MAE, España), y la Vicerrectoría de Investigación, Universidad de Costa Rica (San José, Costa Rica). También se agradece la ayuda del II Plan Andaluz de Investigación (grupo CVI-261).

\section{RESUMEN}

Los euglenoides son eucariotas unicelulares flagelados que habitan ambientes acuáticos y suelos de una gran diversidad de ecosistemas. Este trabajo presenta la descripción morfológica ultraestructural del euglenoide E. pailasensis aislado de las fuentes de lodo caliente de las "pailas de barro" en el Volcán Rincón de la Vieja, Guanacaste, Costa Rica. La temperatura de estos sitios puede variar entre 38 y $98^{\circ} \mathrm{C}$ y puede tener un $\mathrm{pH}$ entre 1 y 4 . El estudio se realizó utilizando microscopia electrónica de barrido y transmisión. El euglenoide está constituido por una célula típica que puede medir de 30 a $45 \mu \mathrm{m}$ de largo y $8-10 \mu \mathrm{m}$ de ancho, con membrana externa, película, cloroplastos, mitocondrias, núcleo, pigmentos granulares y demás organelas citoplasmáticas de un eucarionte. Esta delimitado por una membrana continua y por una película compuesta de aproximadamente 40 - 90 mionemas que miden entre 0.8 y $1.0 \mu \mathrm{m}$ de ancho, dispuestas helicoidalmente sobre la célula. Se observó 5 cloroplastos elongados por célula, de 1-2 $\mu \mathrm{m}$ de diámetro y 6-12 $\mu \mathrm{m}$ de largo, delimitados por tres membranas y localizados en la periferia celular. Este posee glóbulos osmiofílicos y un pirenoide penetrado por pocos tilacoides. El material nutritivo se almacena en gránulos de paramilón y las mitocondrias presentan las crestas en disposición radial hacia el interior del lumen. La zona de la ámpula se observó sin flagelo y se notó una red de fibrillas que envuelve el espécimen. Las características ultraestructurales observadas en este estudio, no permiten explicar la capacidad de E. pailasensis para habitar en el ambiente volcánico extremo de las Pailas de Barro Caliente.

\section{REFERENCIAS}

Adachi, E. 1999. Gallium replication in aquatic and nonaquatic organism scanning electron microscopy. U1tramicroscopy 80: 51-58.

Arnott, H.J. \& M.A. Farmer. 2000. Comparative morphology of the euglenid oellicle. I. Patterns of strips and pores. J. Eukaryot. Microbiol. 47: 469-479.

Brock, T.D. 1986. Introducction: An overview of the thermophiles. pp. 1-16. In: Brock T. D.(eds). Termophi- les, general, molecular and applied microbiology. John Wiley \& Sons, New York.

Dubreuil, R.R. \& G.B. Bouck. 1985. The membrane skeleton of a unicellular organism consists of bridged, articulating strips. J. Cell. Biol. 101: 1884-1896.

Kapfer, M. 1998. Assessment of the colonization and primary production of microphytobenthos in the littoral of acidic mining lakes in Lusatia (Germany). Wat. Air Soil Pollut. 108: 331-340.

Kempner, E.S. \& A.G. Marr. 1970. Growth in volume of Euglena gracilis during the division cycle. J. Bacteriol. 101: 561-567.

Leedale, G.F. 1964. Pellicle structure in Euglena . Brit. Phycol. Bull 2: 291-306.

Leedale, G.F., J.D. Meeuse \& E.G. Pringsheim. 1965. Structure and physiology of Euglena spirogyra. I and II. Arch. für Mikrobiol. 50: 68-102.

Leedale, G.F. 1967. Euglenoid Flagellates. Prentice-Hall. Inc. Englewoud Clifs, N.J. U.S.A. p. 137-192.

Lefort-Tran, M., M.H. Bre, J.L. Ranck \& M. Pouphile. 1980. Euglena plasma membrane during normal and vitamin $\mathrm{B}_{12}$ starvation growth. J. Cell. Sci. 41: 245261.

Lessman, D., R. Deneke, R. Ender, M. Hemm, M. Kapfer, H. Krumbeck, K. Wollmamm \& B. Nixdorf. 1999. Lake Plessa 107 (Lusatia, Germany) - an extremely acidic shallow mining lake. Hidrobiol. 408/409: 293299.

Lowy, J. \& J. Hanson. 1965. Electron microscope studies of bacterial flagella. J. Mol. Biol. 11: 293-313.

Mermelstein, C.S., A. P. Rodríguez, M. Einicker-Lamas, R.E. de B. Navarrete, M. Farina \& M.L. Costa. 1998. Distribution of F-actin, a-actinin, tropomyosin, tubulin and organelles in Euglena gracilis by inmunofluorescence microscopy. Tissue \& Cell 30: 545-553.

Müllner A. N., D. G. Angeler, R. Samuel, E. W. Linton \& R. E. Triemer. 2001. Phylogenetic analysis of phagotropic, phototrophic and osmotrophic euglenoids by using the nuclear 18 sDNA sequence. Int. J. Syst. Evol. Microb. 51: 783-791.

Murray, J.M. 1981. Control of cell shape by calciumin the Euglenophyceae. J. Cell. Sci. 49: 99-117.

Osafune, T., S. Sumida, E., T. Ehara, N. Ueno, E. Hase \& J.A. Schiff. 1990. Lipid (wax) and paramylum as sources of carbon and energy for the early development of proplastidas in dark-grown Euglena gracilis cells transferred to an inorganic medium. J. Electron. Microsc. 39: 372-381. 
Pringsheim, E.G. 1948. Taxonomic problems in the Euglenineae. Biol. Rev. 23: 46-61.

Pringsheim, E.G. 1956. Contribution towards a monograph of the genus Euglena. Nova Acta Leopoldina 18: $1-168$

Schwartzbach, S.D., J.A. Schiff \& N. Goldstein. 1975. Events surrounding the early development of Euglena chloroplasts. Control of paramylum degradation. Plant Physiol. 56: 313-317.

Sittenfeld, A., M. Mora, J.M. Ortega, F. Albertazzi, A. Cordero, M. Roncel, E. Sánchez, M. Vargas, M. Fernández, J. Weckesser \& A. Serrano. 2002. Characterization of a photosynthetic Euglena strain isolated from an acidic hot mud pool of a volcanic area of Costa Rica. FEMS Microbiol. Ecol. 42: 151-161.

Sittenfeld, A., M. Vargas, E. Sánchez, M. Mora \& A. Serrano. 2004. Una nueva especie de Euglena (Euglenozoa: Euglenales) aislada de ambientes extremófilos en las Pailas de Barro del Volcán Rincón de la Vieja, Costa Rica. Rev. Biol. Trop. 52: 27-30.

Sueoka, N., K.S. Chiang \& J.R. Kates. 1967. Deoxiribonucleic acid and replication in meiosis of Chlamydomonas reinhardtii. Isotipic transfer experiments with a strain producing eight zoospores. J. Mol. Biol. 25: 47-66.

Summer, J.R. 1965. The ultrastructure of the pellicle complex of Euglena gracilis. J. Cell. Biol. 24: 253-257.

Van den Hoek, C., D.G. Mann \& H.M. Jahns. 1993. Algae: An introduction to phycology. Cambridge University, Cambridge. 627 p.

Vismara R., L. Barsanti, P. Lupetti, V. Passarelli, D. Mercati, F. Dallai \& P. Gualtieri. 2000. Ultrastructure of the pellicle of Euglena gracilis. Tissue \& Cell 32: 451-456.

Walsh M. M. \& J. Seckbach. 1999. The versatility of microorganisms. p. 153-162. In: Seckbach (eds.). Microorganisms and life in extreme environments. J. Kluver Academic Publishers, Netherlands.

Wen J. 2000. The nuclear matrix of Euglena gracilis (Euglenophyta): A stage of nuclear matrix evolution? Biol. Cell. 92: 125-131.

Zakrys B., J. Cambra-Sanchez \& P.L. Walne. 2001. Chloroplast ultrastructure of Euglena cuneata Pringsheim, E. deses Ehrenberg and E. mutabilis (Euglenophyceae): Taxonomic significance. Acta Protozool. 40: 161-167. 